\title{
Analysis of miRNA expression profiling in human umbilical vein endothelial cells affected by heat stress
}

\author{
JIE LIU ${ }^{1 *}$, GUOGUO ZHU ${ }^{1 *}$, SIYA XU ${ }^{1}$, SHIXIN LIU ${ }^{1}$, QIPING LU $^{2}$ and ZHONGZHI TANG ${ }^{1}$ \\ Departments of ${ }^{1}$ Emergency and ${ }^{2}$ General Surgery, Wuhan General Hospital of People's Liberation Army of China, \\ Wuhan, Hubei 430070, P.R. China
}

Received April 5, 2017; Accepted September 28, 2017

DOI: 10.3892/ijmm.2017.3174

\begin{abstract}
To investigate the regulation of endothelial cell (EC) microRNAs (miRNAs) altered by heat stress, miRNA microarrays and bioinformatics methods were used to determine changes in miRNA profiles and the pathophysiological characteristics of differentially expressed miRNAs. A total of 31 differentially expressed miRNAs were identified, including 20 downregulated and 11 upregulated miRNAs. Gene Ontology (GO) enrichment analysis revealed that the validated targets of the differentially expressed miRNAs were significantly enriched in gene transcription regulation. The pathways were also significantly enriched in the Kyoto Encyclopedia of Genes and Genomes analysis, and most were cancer-related, including the mitogen-activated protein kinase signaling pathway, pathways involved in cancer, the Wnt signaling pathway, the Hippo signaling pathway, proteoglycans involved in cancer and axon guidance. The miRNA-gene and miRNA-GO network analyses revealed several hub miRNAs, genes and functions. Notably, miR-3613-3p played a dominant role in both networks. MAP3K2, MGAT4A, TGFBR1, UBE2R2 and SMAD4 were most likely to be controlled by the altered miRNAs in the miRNA-gene network. The miRNA-GO network analysis revealed significantly complicated associations between miRNAs and different functions, and that the significantly enriched functions targeted by the differentially expressed miRNAs were mostly involved in regulating gene transcription. The present study demonstrated that miRNAs are involved in the pathophysiology of
\end{abstract}

Correspondence to: Dr Qiping Lu, Department of General Surgery, Wuhan General Hospital of People's Liberation Army of China, 627 Wuluo Road, Wuhan, Hubei 430070, P.R. China

E-mail: jw1293913612@163.com

Dr Zhongzhi Tang, Department of Emergency, Wuhan General Hospital of People's Liberation Army of China, 627 Wuluo Road, Wuhan, Hubei 430070, P.R. China

E-mail: 13720335790@163.com

*Contributed equally

Key words: miRNAs, heat stress, endothelial cells, microarray, bioinformatics heat-treated ECs. Understanding the functions of miRNAs may provide novel insights into the molecular mechanisms underlying the heat-induced pathophysiology of ECs.

\section{Introduction}

Heat stroke (HS) is a hyperthermia-associated disease that may induce multi-organ dysfunction and death (1). Abnormalities in endothelial cell (EC) function have been implicated in the pathophysiology of HS (2). EC dysfunction initiates a number of events that trigger EC activation and predispose the vessel wall to increased endothelial permeability, leukocyte adherence, endothelial apoptosis, pro-oxidation and thrombosis (3-9). MicroRNAs (miRNAs) participate in the control of EC-mediated homeostasis $(10,11)$.

miRNAs are small non-coding RNAs of 18-22 nucleotides in length that play an important role in the regulation of gene expression at the post-transcriptional level. Specific classes of miRNAs have been demonstrated to affect the function of ECs. For example, miR-126, miR-128, the miR-17-92 cluster, and the miR-23-24-27 cluster, have been reported to control blood vessel development, growth and differentiation; therefore, these miRNAs and clusters contribute to the regulation of angiogenesis (12-16). Previous studies demonstrated that certain inducible miRNAs also contribute to the regulation of inflammation. Inducible miRNAs, including miR-31, miR-17-3p, miR-126, miR-181b, miR-10a, miR-155, miR-221/222, miR-92, miR-125a/b, miR-365 and miR-663, may participate in the regulation of inflammatory cytokine-induced leukocyte adhesion to ECs and activation of leukocytes (17-27). Recent studies also indicated that certain miRNAs, including miR-155, miR-320, miR-222, miR-125b, miR-410 and miR-218, play an important role in controlling the barrier function of ECs (28-30). These miRNAs reportedly regulate adherens junction disassembly responses, cell migration and cell morphology, which contribute to changes in vascular permeability and integrity. Another set of endothelial miRNAs participate in the control of vascular tone. Several reports have indeed provided evidence that miR-155, miR-221, miR-222, miR-125a/b and miR-21 are associated with the regulation of blood pressure and blood flow by releasing vasodilators or vasoconstrictors (24,31-33). Upon exposure to certain stimuli, ECs may secrete miRNAs that mediate cell-to-cell communication. For example, ECs have 
been shown to produce microvesicles under shear stress. These microvesicles are enriched in miRNAs, particularly miR-143/145 or miR-150, which exert an atheroprotective effect on smooth muscle cells (34-36). This evidence suggests that miRNAs are important molecules that modulate the biological processes of ECs.

Although the involvement of individual miRNAs in endothelial regulation has been characterized in several diseases, changes in the general miRNA expression profiles under heat stress have not been reported. The aim of the present study was to systematically analyze the profiles of miRNA expression in human umbilical vein ECs (HUVECs) exposed to acute heat stress using microarray and bioinformatics analyses, in order to evaluate the potential role of miRNAs in post-transcriptional regulation.

\section{Materials and methods}

HUVEC culture and treatment with heat. HUVECs (American Type Culture Collection, Manassas, VA, USA) were cultured in complete extracellular matrix supplemented with $5 \%$ fetal bovine serum (Invitrogen, Thermo Fisher Scientific, Inc., Waltham, MA, USA), 1\% EC growth supplement (PromoCell, Heidelberg, Germany), $100 \mathrm{U} / \mathrm{ml}$ penicillin and $100 \mu \mathrm{g} / \mathrm{ml}$ streptomycin (Invitrogen, Thermo Fisher Scientific, Inc.), in a humidified atmosphere with $5 \% \mathrm{CO}_{2}$ at $37^{\circ} \mathrm{C}$. All the experiments were performed using 4-6 passages of HUVECs.

In the following experiments, the cells were randomly divided into two groups. The control cells were always placed in an incubator at $37^{\circ} \mathrm{C}$. The cells in the heat treatment group were cultured in the incubator at $43^{\circ} \mathrm{C}$ for $1 \mathrm{~h}$, and then at $37^{\circ} \mathrm{C}$ for a further $24 \mathrm{~h}$.

RNA extraction. Total RNA from each sample was individually isolated using TRIzol (Thermo Fisher Scientific, Inc., Carlsbad, CA, USA) and miRNeasy mini kit (Qiagen, Inc., Valencia, CA, USA) according to the manufacturers' instructions. This procedure efficiently recovered all RNA species, including miRNAs. RNA quantity and quality were measured using a NanoDrop spectrophotometer (ND-1000; NanoDrop Technologies, Wilmington, DE, USA) and RNA integrity was detected by gel electrophoresis.

RNA labeling and array hybridization. The isolated RNA was labeled using the miRCURY' ${ }^{\mathrm{TM}} \mathrm{Hy} 3^{\mathrm{TM}} / \mathrm{Hy} 5^{\mathrm{TM}}$ Power Labeling kit (Exiqon A/S, Vedbaek, Denmark) according to the manufacturer's instructions. Each RNA sample $(1 \mu \mathrm{g})$ was 3'-end-labeled with $\mathrm{Hy}^{\mathrm{TM}}$ fluorescent label, using T4 RNA ligase. Following the array manual, the $\mathrm{Hy}^{\mathrm{TM}}$-labeled samples were then hybridized to the miRCURY ${ }^{\mathrm{TM}}$ LNA array (v.18.0) (Exiqon A/S). The total mixture with hybridization buffer was first denatured for $2 \mathrm{~min}$ at $95^{\circ} \mathrm{C}$, incubated on ice for $2 \mathrm{~min}$, and then hybridized to the microarray for $16-20 \mathrm{~h}$ at $56^{\circ} \mathrm{C}$ in a 12 -Bay Hybridization system (Nimblegen Systems, Inc., Madison, WI, USA). After the hybridization procedure was terminated, the slides were washed several times using the wash buffer kit (Exiqon A/S), and finally dried by centrifugation for $5 \mathrm{~min}$ at $340 \mathrm{x}$ g. The slides were then scanned with the Axon GenePix 4000B microarray scanner (Axon Instruments,
Foster City, CA, USA), which contains 3,100 capture probes annotated in miRBase 18.0, covering all human, mouse and rat miRNAs, as well as all viral miRNAs related to these species. In addition, the microarray scanner contains capture probes for 25 additional new miRPlus ${ }^{\mathrm{TM}}$ human miRNAs that are proprietary and not found in miRBase.

Array data analysis. Scanned images were imported into GenePix Pro 6.0 software (Axon Instruments) for grid alignment and data extraction. Replicated miRNAs were averaged, and miRNAs with intensities $\geq 30$ were selected for calculation of the normalization factor. Expressed data were normalized using the median normalization. Following normalization, significantly differentially expressed miRNAs were identified through volcano plot change filtering. Finally, unsupervised hierarchical clustering was performed based on the normalized data of miRNAs in all samples using Multi Experiment Viewer software, version 4.6 (The Institute for Genomic Research, Rockville, MD, USA). A miRNA was defined as differentially expressed between the compared groups if the $\mathrm{P}$-value was $<0.05$ and the fold change was $>2$.

Reverse transcription-quantitative polymerase chain reaction $(R T-q P C R)$. To validate the authenticity of miRNA expression screened by microarray assay, six differentially expressed miRNAs were analyzed by RT-qPCR. For this analysis, total RNA (10 ng) was reversely transcribed into cDNA using the TaqMan ${ }^{\circledR}$ MicroRNA Reverse Transcription kit (Applied Biosystems, Foster City, CA, USA) according to the manufacturer's instructions. qPCR was performed using SYBR-Green (Thermo Fisher Scientific, Inc.) according to the manufacturer's instructions in the ABI 7300 real-time qPCR system (Bio-Rad Laboratories, Inc., Hercules, CA, USA). The quantification of each miRNA was performed in relation to the U6 housekeeping miRNAs that was used as a standard. The relative abundance of each miRNA was calculated by the comparative $\mathrm{Cq}$ method $\left(2^{-\Delta \Delta \mathrm{Cq}}\right)$, and the results were assessed by using a t-test.

Bioinformatics analysis. Potential target genes of differentially expressed miRNAs were predicted using the three most popular databases, namely, TargetScan, miRanda and miRDB. Only the overlapping genes that were in all three databases were considered as the target genes of the differentially expressed miRNAs. The predicted genes subsequently underwent Gene Ontology (GO) analysis for functional annotation analysis and Kyoto Encyclopedia of Genes and Genomes (KEGG) database analysis to identify the involved enriched pathways. Fisher's exact and Chi-square tests were used to determine the significance of the GO terms and pathways, and the false discovery rate (FDR) was calculated to correct the P-value (37). Only the GO terms and pathways with an adjusted P-value of $<0.05$ and an FDR of $<0.05$ were selected.

miRNA-gene-network analyses. The target genes identified in the functional and pathway enrichment analyses were investigated. The 494 overlapping genes that were in both databases were selected. In addition, to improve the understanding of the associations between the key miRNAs and hub genes, networks for miRNA target genes were constructed according 
TableI.Differentially expressed microRNAs(miRNAs) between heat-treated cells and controls $(\mathrm{P}<0.05$, fold change $>2)$.

\begin{tabular}{|c|c|c|c|}
\hline miRNA & Fold change & $\begin{array}{l}\text { Type of } \\
\text { regulation }\end{array}$ & P-value \\
\hline hsa-miR-4448 & 0.240462612 & Down & $<0.05$ \\
\hline hsa-miR-3180 & 0.243442001 & Down & $<0.05$ \\
\hline hsa-miR-1231 & 0.309725485 & Down & $<0.05$ \\
\hline hsa-miR-3620 & 0.330975034 & Down & $<0.05$ \\
\hline hsa-miR-665 & 0.331296255 & Down & $<0.05$ \\
\hline hsa-miR-4656 & 0.381063941 & Down & $<0.05$ \\
\hline hsa-miR-4458 & 0.384188702 & Down & $<0.05$ \\
\hline hsa-miR-3613-3p & 0.385431663 & Down & $<0.05$ \\
\hline hsa-miR-486-5p & 0.391376978 & Down & $<0.05$ \\
\hline hsa-miR-3180-3p & 0.404438992 & Down & $<0.05$ \\
\hline hsa-miR-378h & 0.416819304 & Down & $<0.05$ \\
\hline hsa-miR-1296 & 0.450624607 & Down & $<0.05$ \\
\hline hsa-miR-616-3p & 0.453329075 & Down & $<0.05$ \\
\hline hsa-miR-4486 & 0.453329201 & Down & $<0.05$ \\
\hline hsa-miR-4749-5p & 0.46624915 & Down & $<0.05$ \\
\hline hsa-miR-489 & 0.468301966 & Down & $<0.05$ \\
\hline hsa-miR-3130-5p & 0.469450744 & Down & $<0.05$ \\
\hline hsa-miR-411-5p & 0.470553479 & Down & $<0.05$ \\
\hline hsa-miR-299-3p & 0.476023136 & Down & $<0.05$ \\
\hline hsa-miR-4665-5p & 0.492758274 & Down & $<0.05$ \\
\hline hsa-miR-1290 & 2.07284178 & Up & $<0.05$ \\
\hline hsa-miR-4430 & 2.135374336 & Up & $<0.05$ \\
\hline hsa-miR-4655-5p & 2.191798238 & Up & $<0.05$ \\
\hline hsa-miR-140-5p & 2.318002303 & Up & $<0.05$ \\
\hline hsa-miR-4500 & 2.324482718 & Up & $<0.05$ \\
\hline hsa-miR-485-3p & 2.399120465 & Up & $<0.05$ \\
\hline hsa-miR-589-5p & 2.399121462 & Up & $<0.05$ \\
\hline hsa-miR-34c-5p & 2.518895027 & Up & $<0.05$ \\
\hline hsa-miR-3158-5p & 3.033915491 & Up & $<0.05$ \\
\hline hsa-miR-3613-5p & 3.690735374 & Up & $<0.05$ \\
\hline hsa-miR-1281 & 4.409294993 & Up & $<0.05$ \\
\hline
\end{tabular}

to the regulatory associations between miRNAs and target genes. The associations of the genes and miRNAs were evaluated by graph theory. The degree was used to measure the regulated effect of miRNAs on genes.

miRNA-GO-network analysis. A miRNA-GO-network was constructed according to the specifically expressed miRNAs and the results of the GO analysis. The control status of GO and miRNAs in the network was evaluated by graph theory, and the degree of miRNAs indicated the number of GO which was regulated by miRNAs in the network. In the same way, a higher degree of GO indicated that more miRNAs contributed to GO.

Statistical analysis. All quantitative data are presented as mean \pm standard deviation. Comparisons between the two groups were performed using the Student's t-test. Comparisons of multiple group data were performed using one-way analysis
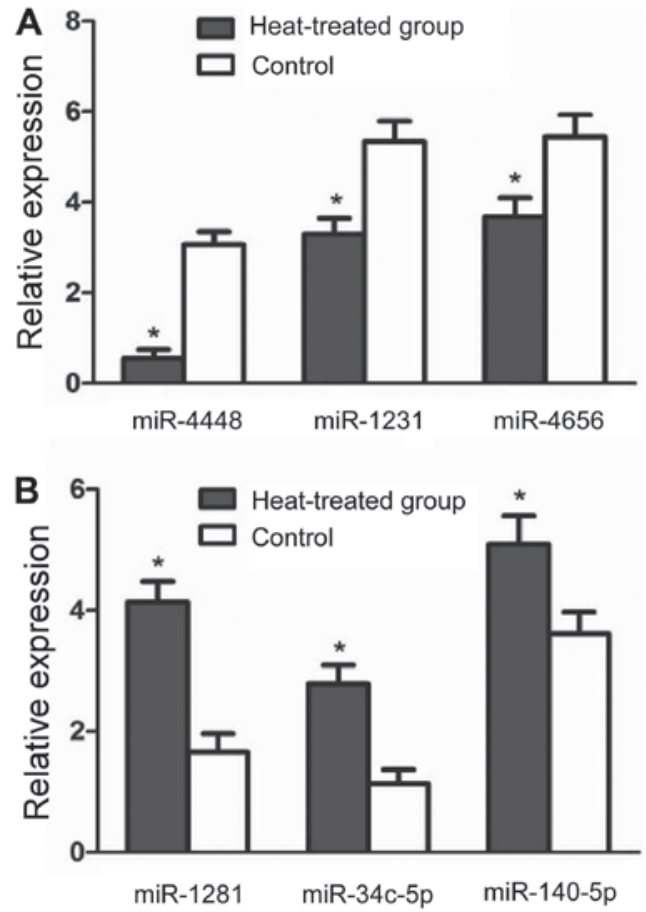

Figure 1. Validation of microRNA (miRNA) microarray results by reverse transcription-quantitative polymerase chain reaction. (A) Downregulated miRNAs in the heat-treated group; (B) upregulated miRNAs in the heat-treated group. All miRNAs exhibited statistically significant changes in expression in the experimental group compared with the control group. ${ }^{*} \mathrm{P}<0.05$

of variance followed by Turkey's post hoc test. P-values $<0.05$ were considered statistically significant. Statistical values were calculated using SPSS software, version 20.0 (IBM Corp., Armonk, NY, USA)

\section{Results}

Screening differentially expressed miRNAs. To identify significantly differentially expressed miRNAs between heat-treated cells and controls, the screening threshold was set to a fold change of $>2$ and a P-value of $<0.05$. In total, 31 miRNAs (20 downregulated and 11 upregulated) were identified (Table I).

Validation by RT-qPCR. Based on their expression levels and fold change in expression, 3 downregulated miRNAs (miR-1281, miR-34c-5p and miR-140-5p) and 3 upregulated miRNAs (miR-4448, miR-1231 and miR-4656) were selected for validation by RT-qPCR. The results revealed a significant intra-group difference in the expression of miRNAs, in a manner consistent with the data obtained from the microarray analysis $(\mathrm{P}<0.05)$ (Fig. 1)

Target gene and bioinformatics analysis. A total of 2,024 target genes were identified for the validated miRNAs. To better understand the potential implications of the differentially expressed miRNAs, these target genes were subjected to $\mathrm{GO}$ analysis to evaluate their potential functions. In the present study, the top GO terms of the target genes of the downregulated miRNAs were transcription (DNA-dependent), regulation of transcription (DNA-dependent), positive 
A

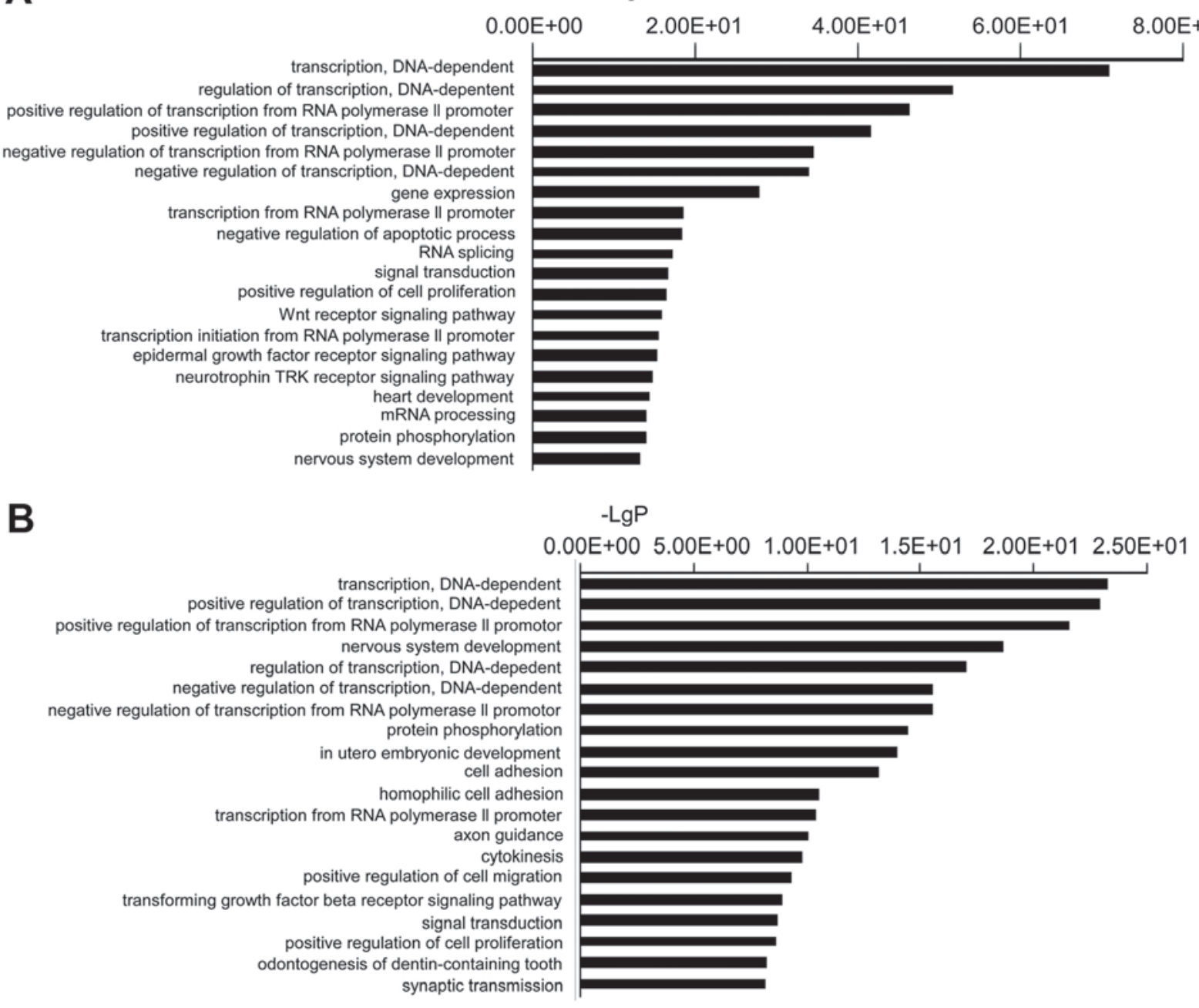

Figure 2. (A) Top 20 functional Gene Ontology (GO) terms of the predicted downregulated microRNA (miRNA) target genes. (B) Top 20 functional GO terms of the predicted upregulated miRNA target genes.

Table II. Crucial microRNAs (miRNAs) in the miRNA-genenetwork (degree $>10$ ).

\section{miRNA}

hsa-miR-3613-3p

hsa-miR-34c-5p

hsa-miR-485-3p

hsa-miR-4458

hsa-miR-4500

hsa-miR-140-5p

hsa-miR-1290

hsa-miR-665

hsa-miR-3158-5p

hsa-miR-3130-5p

hsa-miR-486-5p

hsa-miR-489

hsa-miR-616-3p

hsa-miR-3620

hsa-miR-4448

hsa-miR-378h
Degree regulation of transcription from RNA polymerase II promoter, positive regulation of transcription (DNA-dependent), and negative regulation of transcription from RNA polymerase II promoter. The top GO terms of the target genes of the upregulated miRNAs were transcription (DNA-dependent), positive regulation of transcription (DNA-dependent), positive regulation of transcription from RNA polymerase II promoter, nervous system development, and regulation of transcription (DNA-dependent) (Fig. 2).

The target genes were then subjected to pathway enrichment analysis using KEGG pathways to determine the canonical pathways controlled by the identified miRNAs. Pathways involved in cancer, proteoglycans involved in cancer, the Wnt signaling pathway, axon guidance and Hippo signaling pathways, were the most active pathways regulated by the downregulated miRNAs. The mitogen-activated protein kinase (MAPK) signaling pathway, pathways involved in cancer, the Wnt signaling pathway, the Hippo signaling pathway and proteoglycans involved in cancer, were the top pathways regulated by the upregulated miRNAs (Fig. 3).

miRNA-gene-network analyses. A miRNA-gene-network was constructed according to the results of the GO and pathway 
A

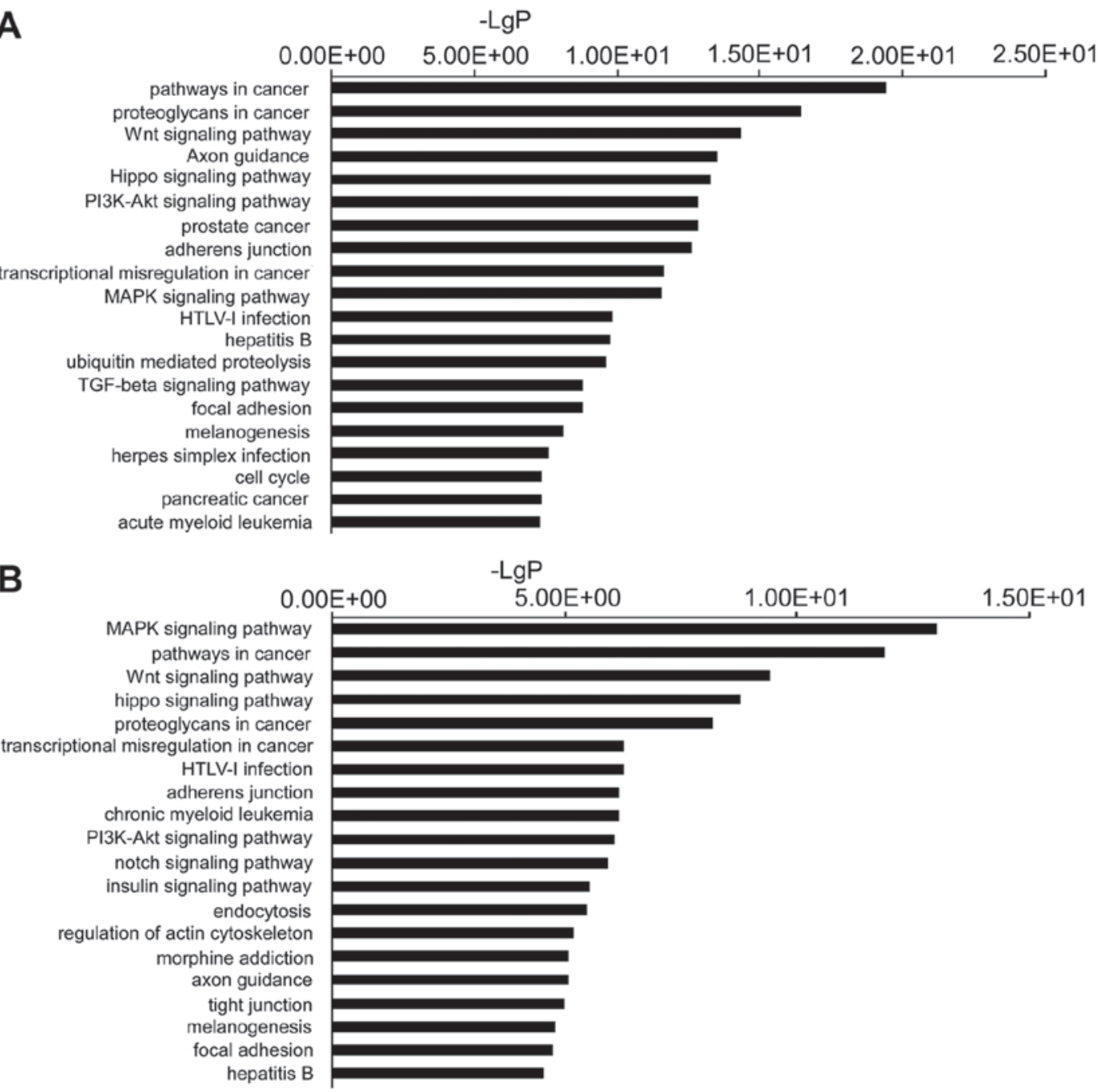

Figure 3. (A) Top 20 signaling pathways predicted to be regulated by downregulated microRNAs (miRNAs). (B) Top 20 signaling pathways predicted to be regulated by upregulated miRNAs. PI3K, phosphoinositide-3 kinase; MAPK, mitogen-activated protein kinase; HTLV, human T-lymphotropic virus; TGF, transforming growth factor.

analyses. The core miRNAs of the interaction network included miR-3613-3p, miR-34c-5p and miR-485-3p (Table II). The network also revealed that MAPK kinase kinase 2 (MAP3K2), $\alpha$-1,3-mannosyl-glycoprotein 4- $\beta$ - $N$-acetylglu cosaminyltransferase A (MGAT4A), transforming growth factor $\beta$ receptor 1 (TGFBR1), ubiquitin-conjugating enzyme E2 R2 (UBE2R2), and Sma- and Mad-related family 4 (SMAD4), were the most crucial target genes (Table III). The associations of miRNAs with genes are shown in Fig. 4.

miRNA-GO-network analysis. The network analysis was useful for determining regulatory associations between the key miRNAs and hub GO. In this network, miR-3613-3p, miR-4458 and miR-4500, which contributed more than the other specifically expressed miRNAs, exhibited 479, 217 and 216 GO functions, respectively (Table IV). The most significantly regulated function-cluster of the total 20 categories was gene expression, whereas others included positive regulation of transcription, positive regulation of transcription by RNA polymerase II promoter and signal transduction (Table V). The significantly complicated associations of the top 20 functions with miRNAs are shown in Fig. 5.

\section{Discussion}

In the present study, the miRNA expression profiles of heattreated HUVECs were evaluated by microarray analysis. A number of miRNAs were obtained from both heat-treated cells and normal controls, and 31 differentially expressed miRNAs were selected, including 20 downregulated and 11 upregulated miRNAs (Table I). The target genes of 31 differentially expressed miRNAs were predicted, and 2,024 target genes were validated. GO and pathway enrichment analysis revealed the significantly different functions and pathways targeted by the differentially expressed miRNAs. A total of 755 notable GO terms and 136 pathways were identified. The most active functions and pathways are shown in Figs. 2 and 3. The wide target range indicates that these miRNAs may play important roles in the biological regulation of HUVECs under heat stress. These results also demonstrated that the differentially expressed miRNAs were predicted to control various functions that were most relevant to the transcription-related process. Moreover, the miRNAs regulated gene expression in ECs under heat treatment via multiple signaling pathways, and biological regulatory mechanisms were integrated in 
Table III. Crucial target genes in the microRNA-gene-network (degree >3).

\begin{tabular}{|c|c|c|}
\hline Gene symbol & Gene description & Degree \\
\hline MAP3K2 & Homo sapiens mitogen-activated protein kinase kinase kinase 2 (MAP3K2), mRNA & 5 \\
\hline MGAT4A & $\begin{array}{l}\text { Homo sapiens mannosyl ( } \alpha \text {-1,3-)-glycoprotein } \beta-1,4-N \text {-acetylglucosaminyltransferase, } \\
\text { isozyme A (MGAT4A), transcript variant } 1, \text { mRNA }\end{array}$ & 4 \\
\hline TGFBR1 & Homo sapiens transforming growth factor, $\beta$ receptor 1 (TGFBR 1$)$, transcript variant 1, mRNA & 4 \\
\hline UBE2R2 & Homo sapiens ubiquitin-conjugating enzyme E2 R2 (UBE2R2), mRNA & 4 \\
\hline SMAD4 & Homo sapiens SMAD family member 4 (SMAD4), mRNA & 4 \\
\hline PRPF40A & $\begin{array}{l}\text { Homo sapiens } \mathrm{PRP} 40 \text { pre-mRNA processing factor } 40 \text { homolog A } \\
\text { (S. cerevisiae) (PRPF40A), mRNA }\end{array}$ & 3 \\
\hline PARVA & Homo sapiens parvin, $\alpha$ (PARVA), mRNA & 3 \\
\hline ACSL4 & $\begin{array}{l}\text { Homo sapiens acyl-CoA synthetase long-chain family member } 4 \text { (ACSL4), } \\
\text { transcript variant } 2 \text {, mRNA }\end{array}$ & 3 \\
\hline ENAH & Homo sapiens enabled homolog (Drosophila) (ENAH), transcript variant 2, mRNA & 3 \\
\hline JAG1 & Homo sapiens jagged 1 (JAG1), mRNA & 3 \\
\hline PDE4D & Homo sapiens phosphodiesterase 4D, cAMP-specific (PDE4D), transcript variant 2, mRNA & 3 \\
\hline E2F5 & Homo sapiens E2F transcription factor 5, p130-binding (E2F5), transcript variant 1, mRNA & 3 \\
\hline DCC & Homo sapiens deleted in colorectal carcinoma (DCC), mRNA & 3 \\
\hline PPM1A & $\begin{array}{l}\text { Homo sapiens protein phosphatase, } \mathrm{Mg}^{2+} / \mathrm{Mn}^{2+} \text {-dependent, } 1 \mathrm{~A}(\mathrm{PPM} 1 \mathrm{~A}) \text {, } \\
\text { transcript variant } 3, \mathrm{mRNA}\end{array}$ & 3 \\
\hline IGF1R & Homo sapiens insulin-like growth factor 1 receptor (IGF1R), mRNA & 3 \\
\hline CDC25A & $\begin{array}{l}\text { Homo sapiens cell division cycle } 25 \text { homolog A (S. pombe) }(\mathrm{CDC} 25 \mathrm{~A}) \text {, } \\
\text { transcript variant } 2 \text {, mRNA }\end{array}$ & 3 \\
\hline CPEB1 & $\begin{array}{l}\text { Homo sapiens cytoplasmic polyadenylation element binding protein } 1 \text { (CPEB1), } \\
\text { transcript variant } 1, \text { mRNA }\end{array}$ & 3 \\
\hline NLK & Homo sapiens nemo-like kinase (NLK), mRNA & 3 \\
\hline FZD3 & Homo sapiens frizzled family receptor 3 (FZD3), transcript variant 2, mRNA & 3 \\
\hline PPP1CB & $\begin{array}{l}\text { Homo sapiens protein phosphatase } 1 \text {, catalytic subunit, } \beta \text { isozyme (PPP1CB), } \\
\text { transcript variant } 3 \text {, mRNA }\end{array}$ & 3 \\
\hline UBOX5 & Homo sapiens U-box domain containing 5 (UBOX5), transcript variant 2, mRNA & 3 \\
\hline BBC3 & $\begin{array}{l}\text { Homo sapiens BCL2-binding component } 3 \text { (BBC3), } \\
\text { nuclear gene encoding mitochondrial protein, transcript variant } 4 \text {, mRNA }\end{array}$ & 3 \\
\hline ACVR1C & Homo sapiens activin A receptor, type IC (ACVR1C), transcript variant 1, mRNA & 3 \\
\hline
\end{tabular}

Table IV. Crucial microRNAs (miRNAs) in the miRNA-GOnetwork (degree $>100)$.

\begin{tabular}{lc}
\hline miRNA & Degree \\
\hline hsa-miR-3613-3p & 479 \\
hsa-miR-4458 & 217 \\
hsa-miR-4500 & 216 \\
hsa-miR-665 & 182 \\
hsa-miR-1290 & 180 \\
hsa-miR-140-5p & 173 \\
hsa-miR-34c-5p & 173 \\
hsa-miR-485-3p & 165 \\
hsa-miR-486-5p & 141 \\
hsa-miR-3158-5p & 135 \\
\hline GO, Gene Ontology. & \\
\hline
\end{tabular}

several main pathways. The majority of the pathways were cancer-related. Following exposure to environmental stressors or cytokines, these pathways may be activated to regulate cell proliferation, migration, differentiation, evasion and angiogenesis.

The network analysis linked the differentially regulated miRNAs to target genes and the relevant biological functions, and facilitated a better understanding of the role of dysregulated miRNAs. In the networks, certain miRNAs functioned as network hubs, such as miR-3613-3p, miR-34c-5p, miR-485-3p, miR-4458 and miR-4500. Of these miRNAs, miR-3613-3p was the most active miRNA in the networks, targeting 237 genes and 479 biological functions (Tables II and IV and Figs. 4 and 5). Previous studies have not focused on miR-3613-3p, and it has not been previously described as participating in EC physiology and pathology. However, plasma miR-3613-3p has been reported as a specific biomarker of lung adenocarcinoma (38). Another study indicated that circulating miR-3613-3p may 


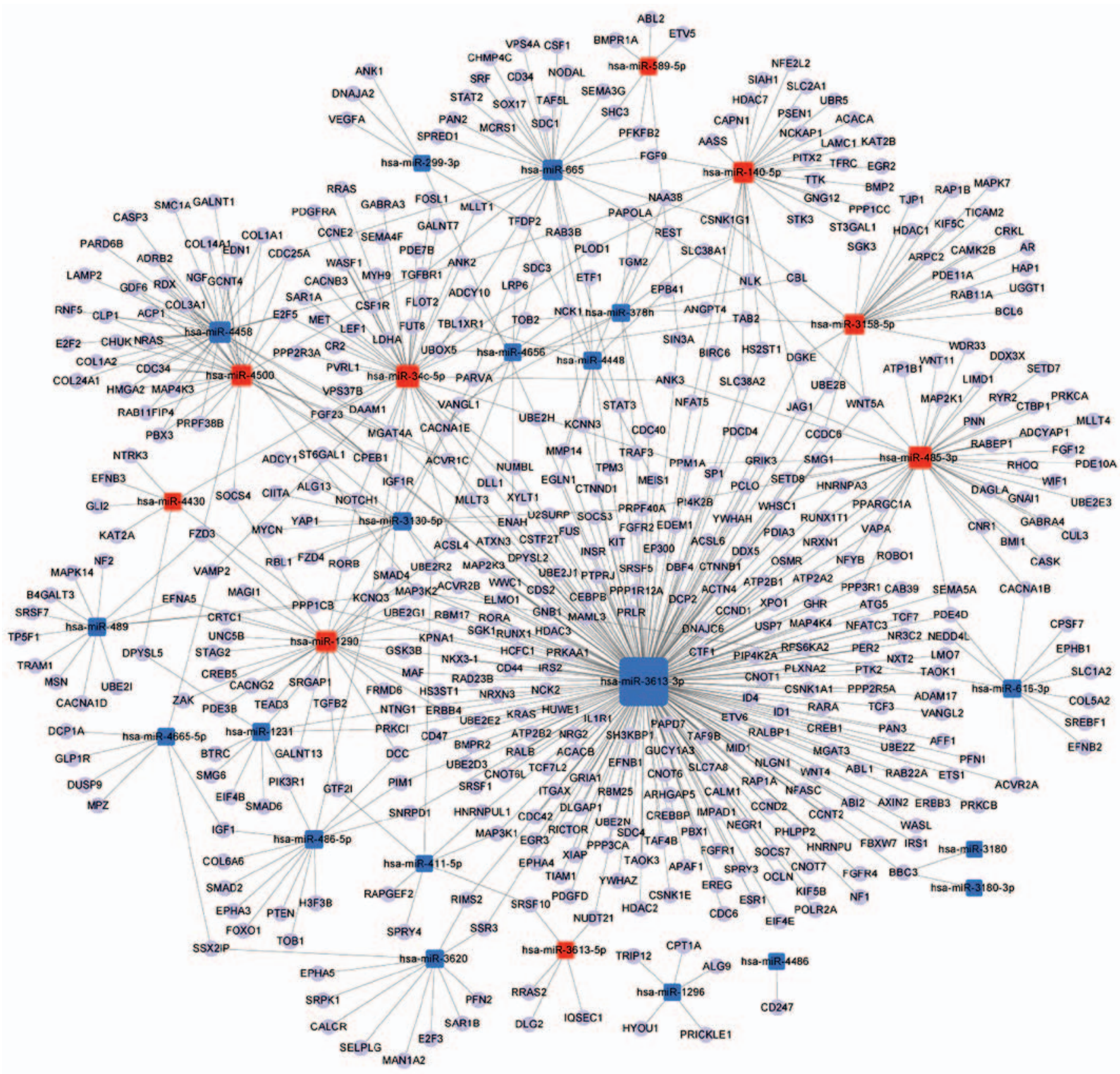

Figure 4. miRNA-gene-network showing the associations between key microRNAs (miRNAs) and the relevant hub genes. The square nodes represent miRNAs (red nodes, upregulated miRNAs; blue nodes, downregulated miRNAs), and the circular nodes represent target genes. The lines indicate the regulatory effect of miRNAs on target genes. The size of the square nodes indicates the degree to which miRNAs contribute to the network.

predict persistent severe axial pain following exposure to common stressors, and may be involved in the pathogenesis of post-traumatic musculoskeletal pain (39). Liu et al found that miR-3613-3p was upregulated in left atrial appendages in atrial fibrillation (40). Moreover, Wang et al reported that urinary expression of miR-3613-3p was downregulated in patients with immunoglobulin A nephropathy, and the levels of miR-3613-3p were correlated with disease severity (41). Therefore, although the specific mechanism of function of miR-3613-3 remains unknown, decreased miR-3613-3p expression may exert a modulatory effect on ECs under heat stress. Additional studies are required to elucidate the detailed role of miR-3613-3p in ECs.
The miRNA-gene network analysis revealed a range of hub genes, such as MAP3K2, MGAT4A, TGFBR1, UBE2R2 and SMAD4 (Table III). MAP3K2 encodes MAPK kinase kinase 2 (MEKK2), which is a component of a protein kinase signal transduction cascade that preferentially regulates the c-Jun N-terminal kinases (JNKs) and extracellular signal-regulated kinase 5 (ERK5) pathways by phosphorylating and activating MAP2K5 as well as MAP2K7 in a similar manner $(42,43)$. The MAP2K5/ERK5 pathway is required for normal cardiovascular development and vascular integrity, improves EC viability and reduces apoptosis $(44,45)$, whereas JNK MAPKs promote apoptosis of ECs under most conditions (46). MEKK2 coordinately activates signaling through 
Table V. Crucial GO functions in the microRNA-GO-network (degree $>15$ ).

GO (name)

Degree

Gene expression

Positive regulation of transcription, DNA-dependent

Positive regulation of transcription from RNA polymerase II promoter

Signal transduction

Negative regulation of transcription from RNA polymerase II promoter

Small molecule metabolic process

Transforming growth factor- $\beta$ receptor signaling pathway

Axon guidance

Innate immune response

Transcription, DNA-dependent

Virus-host interaction

Negative regulation of apoptotic process

Negative regulation of transcription, DNA-dependent

Regulation of transcription, DNA-dependent

Synaptic transmission

Transcription initiation from RNA polymerase II promoter 16

Heart development

Mitotic cell cycle

Positive regulation of cell proliferation

Protein phosphorylation

GO, Gene Ontology.

MEK5/ERK5 and MEK7/JNK protein kinases to respond to different stimuli. MEKK2 has also been shown to directly phosphorylate and activate $\mathrm{I} \kappa \mathrm{B}$ kinases and, thus, plays a role in the nuclear factor- $\kappa \mathrm{B}(\mathrm{NF}-\kappa \mathrm{B})$ signaling pathway $(47,48)$. MEKK2 has also been found to bind and activate protein kinase C-related kinase 2, which suggests its involvement in a regulated signaling process (49). MGAT4A encodes the glycosyltransferase $N$-acetylglucosaminyltransferase IVa, and catalyzes the transfer of $N$-acetylglucosamine (GlcNAc) from UDP-GlcNAc to the GlcNAc $\beta 1$-2Mana1-3 arm of the $N$-glycan core via a $\beta 1-4$ linkage $(50,51)$. As an important post-translational modification of target proteins, $\mathrm{N}$-glycosylation plays an important role in cell proliferation, differentiation, apoptosis and development, during embryogenesis as well as in mature tissues (52-54). The functions of $N$-glycans associated with cell surface receptors are of great importance, since $N$-glycans are directly involved in controlling cellular functions. The target proteins of glycosyltransferases include epidermal growth factor receptor, human epidermal growth factor receptor 3 , transforming growth factor- $\beta$ (TGF- $\beta$ ) receptor, T-cell receptors, $\beta$-site APP cleaving enzyme 1 , glucose transporter 2 , E-cadherin and $\alpha 5 \beta 1$ integrin (52). TGFBR1 encodes TGF- $\beta$ type I receptor $(T \beta R I)$, which is the sole known cell surface receptor serine/threonine kinase in humans and participates in TGF- $\beta$ signaling. The binding of TGF- $\beta$ to T $\beta$ RII dimers stabilizes the interaction of T $\beta$ RII dimers with two T $\beta$ RI molecules to form a ligand receptor-complex involving a heterotetrameric receptor and a ligand dimer (55). Ligand binding to T $\beta$ RII causes the phosphorylation of T $\beta$ RI, which activates Smad2 and Smad3 (55). The activated Smad proteins form complexes with Smad4 and are translocated into the nucleus, where they regulate the transcription of target genes $(56,57)$. TGF- $\beta 1$ exerts its effects on a variety of cells through activation of the Smad proteins and other signaling pathways, including MAPKs, small GTPases, protein kinase C and phosphoinositide 3-kinase $(58,59)$. Under most conditions, the activation of the TGF- $\beta 1$ signaling pathway inhibits EC proliferation and migration and induces EC apoptosis (60). Smad4 is a central mediator of TGF- $\beta 1 / \mathrm{Smad}$ signaling. Smad4 belongs to the evolutionarily conserved family of Smad proteins that are crucial for transmitting TGF- $\beta 1$ superfamily signals from the cell surface to the nucleus (61). UBE2R is involved in the ubiquitin cascade. The ubiquitin-proteasome system (UPS) comprises ubiquitin, ubiquitin-activating enzyme (E1), ubiquitin-binding enzyme (E2), ubiquitinligating enzymes (E3) and the $26 \mathrm{~S}$ proteasome (62). Ubiquitin is attached to substrates by a complex three-step enzymatic cascade, which involves E1 ubiquitin activation $(62,63)$, E2 ubiquitin conjugation and a variety of E3 ubiquitin-ligating enzymes $(64,65)$. The UPS plays an important role in the regulation of cellular processes via degradation of damaged proteins and the control of protein quantity in cells $(66,67)$. Although the common effect of ubiquitylation is degradation, the degradation-independent Ub-signaling system also affects various biological processes, which include cytokine production, proliferation, apoptosis, inflammation, canalization, pain sensitization to host defense by regulation of protein kinase activation, signal transduction, membrane transport, transcriptional regulation, gene silencing, DNA injury repair and DNA replication $(62,68)$. All these genes receive physicochemical 


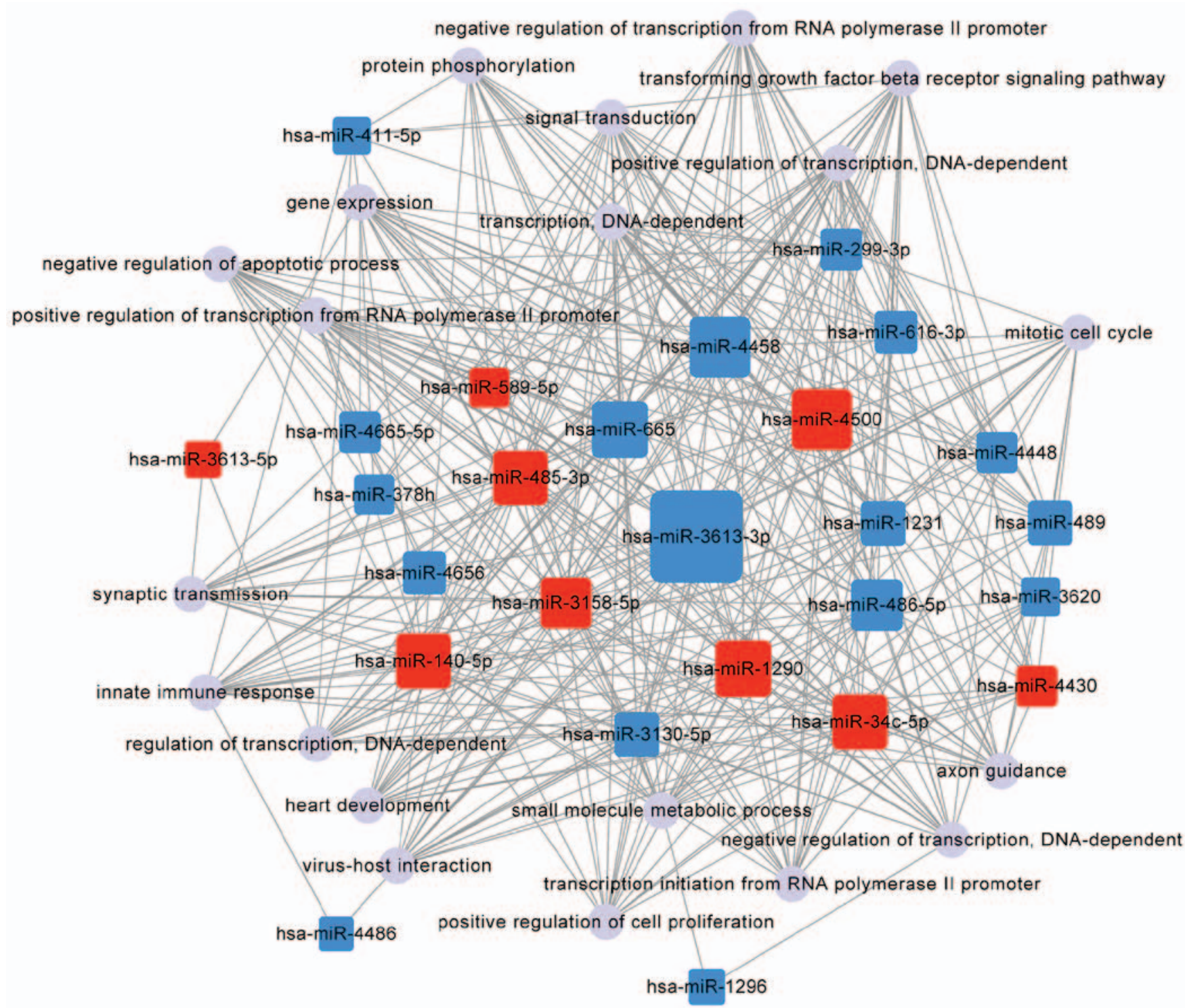

Figure 5. Interaction between the top 20 targeted functions and the differentially expressed microRNAs (miRNAs). The red square nodes represent upregulated miRNAs, the blue square nodes represent downregulated miRNAs, and the violet circular nodes represent target functions. The lines represent the inhibitory effect of miRNAs on target functions. The size of square nodes indicates the degree to which the miRNAs contribute to the network.

stimuli and inputs from a diverse array of receptors and coordinate the activation of multiple responses.

In addition to the miRNA-gene network analysis, the miRNA-GO network analysis provides a more detailed view of the integrated association between the regulation of miRNAs and heat stress. Among the top 10 functions, gene expression was the most active and was regulated by 21 differentially expressed miRNAs. Furthermore, 4 transcription-related functions were regulated by 78 differentially expressed miRNAs (Table V). These findings indicated that heat stress may affect EC gene expression directly via differentially expressed miRNAs, or induce alterations in transcriptionrelated functions indirectly via miRNA profile changes. This finding was not surprising, as most of the hub genes participate in gene expression and transcription via certain downstream molecules. For example, MAP3K2 was one of the top genes revealed by the miRNA-gene network. The most significant role of ERK5, which is a downstream target of MAP3K2, appears to be the regulation of a number of downstream transcription factors, including the myocyte-enhancer factor (MEF) family, MEF2A, C and D, which are the best characterized members of this family $(69,70)$. ERK5 also directly enhances the transcription of c-Myc, cAMP-response element-binding protein, and Sapla (71-73). Enhancement of Sapla occurs via a serum response element that may also be involved in activating the c-Fos promoter (74). JNKs, another target of MAP3K2, translocate from the cytoplasm to the nucleus once activated, and regulate a series of transcription factors, such as c-Jun, Ets-like protein-1, activating transcription factor 2, p53, c-Myc, and nuclear factor of activated T-cells (75-79).

In the present study, the miRNA-GO network analysis revealed a significantly complicated association between miRNAs and different functions (Fig. 5 and Tables IV and V). First, more functions than genes that were targeted by miRNAs were found. Second, each differentially expressed miRNA modulated a large variety of functions, and each function was controlled by several miRNAs, thus resulting in a complicated network. For example, in the present study, 
miR-3613-3p targeted 479 functions, and the function of gene expression was regulated by 21 differentially expressed miRNAs (Tables IV and V). Third, one miRNA may simultaneously regulate opposing functions. For example, certain miRNAs, such as miR-3613-3p, miR-4458 or miR-4500, were not only involved in the positive regulation of transcription, but also in the negative regulation of transcription (data not shown). Finally, certain miRNAs contributed to the positive regulatory function, and other miRNAs contributed to the corresponding negative regulatory function. For example, miR-665 participated in the negative regulation of the apoptotic process, but miR-4500 not only participated in the anti-apoptotic process, but also promoted the cellular apoptosis process (data not shown). Moreover, each function was affected by both upregulated and downregulated miRNAs (data not shown). Our results suggested that the functions regulated by miRNAs were not an isolated event, and this regulation depends on the balance between the miRNAs that promote the function and the miRNAs that suppress the function. The miRNA-GO network provided a global view of the molecular pathophysiology of EC heat stress and helped identify the key 'players' in the whole network.

There were several limitations to this study. First, we were limited by the use of HUVECs. Although the baseline miRNA expression levels are similar between ECs from different vascular beds, some unique miRNA phenotypic diversity is present among the different types of ECs (80). As only one cell type was assessed, 'cell-specific' miRNAs could not be identified in our analysis, which could be determined using other cell types. Second, the number of samples utilized for the miRNA microarray was relatively small and, therefore, less predominantly differentiated miRNAs were difficult to detect with the microarrays due to low power. Third, the miRNA microarray technique had certain limitations, as some of the microarray results may have been affected by technical artifacts introduced by sample selection, the hybridization procedure, or the microarray platform.

In conclusion, this study demonstrated that 31 miRNAs were differentially expressed in ECs under heat stress. The bioinformatics analysis predicted the target genes, functions and signaling pathways of these miRNAs. The miRNA-gene and miRNA-GO network analyses revealed a range of hub miRNAs, genes and functions, which may consolidate our understanding of the involvement of miRNAs in the pathophysiology of heat-treated ECs. miR-3613-3p may be involved in heat stress by regulating relevant genes and functions. Although further mechanistic investigation is required, the findings presented herein provide novel insights into the molecular mechanisms of heat stress in ECs.

\section{References}

1. Bouchama A and Knochel JP: Heat stroke. N Engl J Med 346 1978-1988, 2002.

2. Roberts GT, Ghebeh H, Chishti MA, Al-Mohanna F, El-Sayed R, Al-Mohanna $\mathrm{F}$ and Bouchama A: Microvascular injury, thrombosis, inflammation, and apoptosis in the pathogenesis of heatstroke: A study in baboon model. Arterioscler Thromb Vasc Biol 28: 1130-1136, 2008

3. Bazzoni G and Dejana E: Endothelial cell-to-cell junctions: Molecular organization and role in vascular homeostasis. Physiol Rev 84: 869-901, 2004.
4. Busse R and Fleming I: Vascular endothelium and blood flow. Handb Exp Pharmacol 176: 43-78, 2006.

5. Toda N, Nakanishi S and Tanabe S: Aldosterone affects blood flow and vascular tone regulated by endothelium-derived NO: Therapeutic implications. Br J Pharmacol 168: 519-533, 2013.

6. Cines DB, Pollak ES, Buck CA, Loscalzo J, Zimmerman GA, McEver RP, Pober JS, Wick TM, Konkle BA, Schwartz BS, et al: Endothelial cells in physiology and in the pathophysiology of vascular disorders. Blood 91: 3527-3561, 1998.

7. Michiels C: Endothelial cell functions. J Cell Physiol 196: 430-443, 2003.

8. Minshall RD and Malik AB: Transport across the endothelium: Regulation of endothelial permeability. Handb Exp Pharmacol 176: 107-144, 2006.

9. Pober JS and Sessa WC: Evolving functions of endothelial cells in inflammation. Nat Rev Immunol 7: 803-815, 2007.

10. Santoro MM and Nicoli S: miRNAs in endothelial cell signaling: The endomiRNAs. Exp Cell Res 319: 1324-1330, 2013.

11. Staszel T, Zapała B, Polus A, Sadakierska-Chudy A, Kieć-Wilk B, Stępień E, Wybrańska I, Chojnacka M and Dembińska-Kieć A: Role of microRNAs in endothelial cell pathophysiology. Pol Arch Med Wewn 121: 361-366, 2011.

12. Wang S, Aurora AB, Johnson BA, Qi X, McAnally J, Hill JA, Richardson JA, Bassel-Duby R and Olson EN: The endothelialspecific microRNA miR-126 governs vascular integrity and angiogenesis. Dev Cell 15: 261-271, 2008.

13. Small EM, Sutherland LB, Rajagopalan KN, Wang $S$ and Olson EN: MicroRNA-218 regulates vascular patterning by modulation of Slit-Robo signaling. Circ Res 107: 1336-1344, 2010.

14. Doebele C, Bonauer A, Fischer A, Scholz A, Reiss Y, Urbich C, Hofmann WK, Zeiher AM and Dimmeler S: Members of the microRNA-17-92 cluster exhibit a cell-intrinsic antiangiogenic function in endothelial cells. Blood 115: 4944-4950, 2010.

15. Qin X, Wang X, Wang Y, Tang Z, Cui Q, Xi J, Li YS, Chien S and Wang N: MicroRNA-19a mediates the suppressive effect of laminar flow on cyclin D1 expression in human umbilical vein endothelial cells. Proc Natl Acad Sci USA 107: 3240-3244, 2010.

16. Bang C, Fiedler J and Thum T: Cardiovascular importance of the microRNA-23/27/24 family. Microcirculation 19: 208-214, 2012.

17. O'Connell RM, Taganov KD, Boldin MP, Cheng G and Baltimore D: MicroRNA-155 is induced during the macrophage inflammatory response. Proc Natl Acad Sci USA 104: 1604-1609, 2007.

18. Tili E, Michaille JJ, Cimino A, Costinean S, Dumitru CD, Adair B, Fabbri M, Alder H, Liu CG, Calin GA, et al: Modulation of miR-155 and miR-125b levels following lipopolysaccharide/ TNF-alpha stimulation and their possible roles in regulating the response to endotoxin shock. J Immunol 179: 5082-5089, 2007.

19. Sun X, Icli B, Wara AK, Belkin N, He S, Kobzik L, Hunninghake GM, Vera MP, Blackwell TS, Baron RM, et al; MICU Registry: MicroRNA-181b regulates NF- $\kappa \mathrm{B}$-mediated vascular inflammation. J Clin Invest 122: 1973-1990, 2012.

20. Fang Y, Shi C, Manduchi E, Civelek M and Davies PF: MicroRNA-10a regulation of proinflammatory phenotype in athero-susceptible endothelium in vivo and in vitro. Proc Nat Acad Sci USA 107: 13450-13455, 2010.

21. Qin B, Xiao B, Liang D, Xia J, Li Y and Yang H: MicroRNAs expression in ox-LDL treated HUVECs: MiR-365 modulates apoptosis and Bcl-2 expression. Biochem Biophys Res Commun 410: 127-133, 2011.

22. Fang Y and Davies PF: Site-specific microRNA-92a regulation of Kruppel-like factors 4 and 2 in atherosusceptible endothelium. Arterioscler Thromb Vasc Biol 32: 979-987, 2012.

23. Zhu N, Zhang D, Chen S, Liu X, Lin L, Huang X, Guo Z, Liu J, Wang Y, Yuan W, et al: Endothelial enriched microRNAs regulate angiotensin II-induced endothelial inflammation and migration. Atherosclerosis 215: 286-293, 2011.

24. Li D, Yang P, Xiong Q, Song X, Yang X, Liu L, Yuan W and Rui YC: MicroRNA-125a/b-5p inhibits endothelin-1 expression in vascular endothelial cells. J Hypertens 28: 1646-1654, 2010.

25. Ni CW, Qiu H and Jo H: MicroRNA-663 upregulated by oscillatory shear stress plays a role in inflammatory response of endothelial cells. Am J Physiol Heart Circ Physiol 300: H1762-H1769, 2011.

26. Suárez Y, Wang C, Manes TD and Pober JS: Cutting edge: TNF-induced microRNAs regulate TNF-induced expression of E-selectin and intercellular adhesion molecule-1 on human endothelial cells: feedback control of inflammation. J Immunol 184: 21-25, 2010. 
27. Harris TA, Yamakuchi M, Ferlito M, Mendell JT and Lowenstein CJ: MicroRNA-126 regulates endothelial expression of vascular cell adhesion molecule 1. Proc Natl Acad Sci USA 105: $1516-1521,2008$

28. Dejana E, Corada M and Lampugnani MG: Endothelial cell-tocell junctions. FASEB J 9: 910-918, 1995.

29. Pepini T, Gorbunova EE, Gavrilovskaya IN, Mackow JE and Mackow ER: Andes virus regulation of cellular microRNAs contributes to hantavirus-induced endothelial cell permeability. J Virol 84: 11929-11936, 2010.

30. Nicoloso MS, Spizzo R, Shimizu M, Rossi S and Calin GA MicroRNAs - the micro steering wheel of tumour metastases Nat Rev Cancer 9: 293-302, 2009.

31. Sun HX, Zeng DY, Li RT, Pang RP, Yang H, Hu YL, Zhang Q Jiang Y, Huang LY, Tang YB, et al: Essential role of microRNA-155 in regulating endothelium-dependent vasorelaxation by targeting endothelial nitric oxide synthase. Hypertension 60: 1407-1414, 2012.

32. Suárez Y, Fernández-Hernando C, Pober JS and Sessa WC: Dicer dependent microRNAs regulate gene expression and functions in human endothelial cells. Circ Res 100: 1164-1173, 2007.

33. Weber M,Baker MB, Moore JP and Searles CD: MiR-21 is induced in endothelial cells by shear stress and modulates apoptosis and eNOS activity. Biochem Biophys Res Commun 393: 643-648, 2010.

34. Hergenreider E, Heydt S, Tréguer K, Boettger T, Horrevoets AJ, Zeiher AM, Scheffer MP, Frangakis AS, Yin X, Mayr M, et al: Atheroprotective communication between endothelial cells and smooth muscle cells through miRNAs. Nat Cell Biol 14 249-256, 2012

35. Zhang Y, Liu D, Chen X, Li J, Li L, Bian Z, Sun F, Lu J, Yin Y, Cai X, et al: Secreted monocytic miR-150 enhances targeted endothelial cell migration. Mol Cell 39: 133-144, 2010.

36. Chamorro-Jorganes A, Araldi E and Suárez Y: MicroRNAs as pharmacological targets in endothelial cell function and dysfunction. Pharmacol Res 75: 15-27, 2013

37. Benjamini Y, Drai D, Elmer G, Kafkafi N and Golani I: Controlling the false discovery rate in behavior genetics research. Behav Brain Res 125: 279-284, 2001.

38. Pu Q, Huang Y, Lu Y, Peng Y, Zhang J, Feng G, Wang C, Liu L and Dai Y: Tissue-specific and plasma microRNA profiles could be promising biomarkers of histological classification and TNM stage in non-small cell lung cancer. Thorac Cancer 7: 348-354, 2016.

39. Linnstaedt SD, Walker MG, Parker JS, Yeh E, Sons RL, Zimny E, Lewandowski C, Hendry PL, Damiron K, Pearson C, et al: MicroRNA circulating in the early aftermath of motor vehicle collision predict persistent pain development and suggest a role for microRNA in sex-specific pain differences. Mol Pain 11: 66, 2015.

40. Liu H, Chen GX, Liang MY, Qin H, Rong J, Yao JP and Wu ZK: Atrial fibrillation alters the microRNA expression profiles of the left atria of patients with mitral stenosis. BMC Cardiovasc Disord 14: 10, 2014

41. Wang N, Bu R, Duan Z, Zhang X, Chen P, Li Z, Wu J, Cai G and Chen $X$ : Profiling and initial validation of urinary microRNAs as biomarkers in IgA nephropathy. PeerJ 3: e990, 2015.

42. Dermott JM, Ha JH, Lee CH and Dhanasekaran N: Differential regulation of Jun N-terminal kinase and p38MAP kinase by Galpha12. Oncogene 23: 226-232, 2004.

43. Raviv Z, Kalie E and Seger R: MEK5 and ERK5 are localized in the nuclei of resting as well as stimulated cells, while MEKK2 translocates from the cytosol to the nucleus upon stimulation. J Cell Sci 117: 1773-1784, 2004.

44. Nithianandarajah-Jones GN, Wilm B, Goldring CE, Müller J and Cross MJ: The role of ERK5 in endothelial cell function. Biochem Soc Trans 42: 1584-1589, 2014.

45. Wu Y and Chakrabarti S: ERK5 mediated signalling in diabetic retinopathy. Med Hypothesis Discov Innov Ophthalmol 4: 17-26, 2015.

46. Weston CR and Davis RJ: The JNK signal transduction pathway. Curr Opin Cell Biol 19: 142-149, 2007.

47. Schmidt C, Peng B, Li Z, Sclabas GM, Fujioka S, Niu J, Schmidt-Supprian M, Evans DB, Abbruzzese JL and Chiao PJ: Mechanisms of proinflammatory cytokine-induced biphasic NF-kappaB activation. Mol Cell 12: 1287-1300, 2003.

48. Winsauer G, Resch U, Hofer-Warbinek R, Schichl YM and de Martin R: XIAP regulates bi-phasic NF-kappaB induction involving physical interaction and ubiquitination of MEKK2 Cell Signal 20: 2107-2112, 2008.
49. Sun W, Vincent S, Settleman J and Johnson GL: MEK kinase 2 binds and activates protein kinase C-related kinase 2 . Bifurcation of kinase regulatory pathways at the level of an MAPK kinase kinase. J Biol Chem 275: 24421-24428, 2000.

50. Oguri S, Yoshida A, Minowa MT and Takeuchi M: Kinetic properties and substrate specificities of two recombinant human $N$-acetylglucosaminyltransferase-IV isozymes. Glycoconj J 23: 473-480, 2006

51. Minowa MT, Oguri S, Yoshida A, Hara T, Iwamatsu A, Ikenaga $\mathrm{H}$ and Takeuchi M: cDNA cloning and expression of bovine UDP- $N$-acetylglucosamine: alpha1, 3-D-mannoside beta1,4- $N$ acetylglucosaminyltransferase IV. J Biol Chem 273: 11556-11562, 1998.

52. Takahashi M, Kizuka Y, Ohtsubo K, Gu J and Taniguchi N: Disease-associated glycans on cell surface proteins. Mol Aspects Med 51: 56-70, 2016

53. Fan J, Wang S, Yu S, He J, Zheng W and Zhang J: $N$-acetylglucosaminyltransferase IVa regulates metastatic potential of mouse hepatocarcinoma cells through glycosylation of CD147. Glycoconj J 29: 323-334, 2012.

54. Niimi K, Yamamoto E, Fujiwara S, Shinjo K, Kotani T, Umezu T, Kajiyama H, Shibata K, Ino K and Kikkawa F: High expression of $N$-acetylglucosaminyltransferase IVa promotes invasion of choriocarcinoma. Br J Cancer 107: 1969-1977, 2012.

55. López-Casillas F, Wrana JL and Massagué J: Betaglycan presents ligand to the TGF beta signaling receptor. Cell 73: 1435-1444, 1993.

56. Yin JJ, Selander K, Chirgwin JM, Dallas M, Grubbs BG, Wieser R, Massagué J, Mundy GR and Guise TA: TGF-beta signaling blockade inhibits PTHrP secretion by breast cancer cells and bone metastases development. J Clin Invest 103: 197-206, 1999.

57. Siegel PM and Massagué J: Cytostatic and apoptotic actions of TGF-beta in homeostasis and cancer. Nat Rev Cancer 3: 807-821, 2003.

58. Smith AL, Iwanaga R, Drasin DJ, Micalizzi DS, Vartuli RL, Tan AC and Ford HL: The miR-106b-25 cluster targets Smad7, activates TGF- $\beta$ signaling, and induces EMT and tumor initiating cell characteristics downstream of Six1 in human breast cancer. Oncogene 31: 5162-5171, 2012

59. Chu IM, Lai WC, Aprelikova O, El Touny LH, Kouros-Mehr H and Green JE: Expression of GATA3 in MDA-MB-231 triplenegative breast cancer cells induces a growth inhibitory response to TGF $\beta$. PLoS One 8: e61125, 2013.

60. Roberts AB and Sporn MB: Regulation of endothelial cell growth, architecture, and matrix synthesis by TGF-beta. Am Rev Respir Dis 140: 1126-1128, 1989.

61. Zhang Y, Feng XH and Derynck R: Smad3 and Smad4 cooperate with c-Jun/c-Fos to mediate TGF-beta-induced transcription. Nature 394: 909-913, 1998

62. Swatek KN and Komander D: Ubiquitin modifications. Cell Res 26: 399-422, 2016.

63. Schulman BA and Harper JW: Ubiquitin-like protein activation by E1 enzymes: The apex for downstream signalling pathways. Nat Rev Mol Cell Biol 10: 319-331, 2009.

64. Ye Y and Rape M: Building ubiquitin chains: E2 enzymes at work. Nat Rev Mol Cell Biol 10: 755-764, 2009.

65. Deshaies RJ and Joazeiro CA: RING domain E3 ubiquitin ligases. Annu Rev Biochem 78: 399-434, 2009.

66. Komander D and Rape M: The ubiquitin code. Annu Rev Biochem 81: 203-229, 2012

67. Hoeller D, Hecker CM and Dikic I: Ubiquitin and ubiquitin-like proteins in cancer pathogenesis. Nat Rev Cancer 6: 776-788, 2006.

68. Meier P, Morris O and Broemer M: Ubiquitin-mediated regulation of cell death, inflammation, and defense of homeostasis. Curr Top Dev Biol 114: 209-239, 2015.

69. Yang CC, Ornatsky OI, McDermott JC, Cruz TF and Prody CA Interaction of myocyte enhancer factor 2 (MEF2) with a mitogenactivated protein kinase, ERK5/BMK1. Nucleic Acids Res 26: 4771-4777, 1998

70. Kato Y, Zhao M, Morikawa A, Sugiyama T, Chakravortty D, Koide N, Yoshida T, Tapping RI, Yang Y, Yokochi T, et al: Big mitogen-activated kinase regulates multiple members of the MEF2 protein family. J Biol Chem 275: 18534-18540, 2000.

71. Kamakura S, Moriguchi T and Nishida E: Activation of the protein kinase ERK5/BMK1 by receptor tyrosine kinases. Identification and characterization of a signaling pathway to the nucleus. J Biol Chem 274: 26563-26571, 1999.

72. English JM, Pearson G, Baer R and Cobb MH: Identification of substrates and regulators of the mitogen-activated protein kinase ERK5 using chimeric protein kinases. J Biol Chem 273: 3854-3860, 1998 . 
73. Watson FL, Heerssen HM, Bhattacharyya A, Klesse L, Lin MZ and Segal RA: Neurotrophins use the Erk5 pathway to mediate a retrograde survival response. Nat Neurosci 4: 981-988, 2001.

74. Nithianandarajah-Jones GN, Wilm B, Goldring CE, Müller J and Cross MJ: ERK5: Structure, regulation and function. Cell Signal 24: 2187-2196, 2012.

75. McCubrey JA, Steelman LS, Chappell WH, Abrams SL, Wong EW, Chang F, Lehmann B, Terrian DM, Milella M, Tafuri A, et al: Roles of the Raf/MEK/ERK pathway in cell growth, malignant transformation and drug resistance. Biochim Biophys Acta 1773. 1263-1284, 2007.

76. Oleinik NV, Krupenko NI and Krupenko SA: Cooperation between JNK1 and JNK2 in activation of p53 apoptotic pathway. Oncogene 26: 7222-7230, 2007.

77. Iavarone C, Catania A, Marinissen MJ, Visconti R, Acunzo M, Tarantino C, Carlomagno MS, Bruni CB, Gutkind JS and Chiariello M: The platelet-derived growth factor controls c-myc expression through a JNK- and AP-1-dependent signaling pathway. J Biol Chem 278: 50024-50030, 2003.
78. Zhong S, Fromm J and Johnson DL: TBP is differentially regulated by c-Jun N-terminal kinase 1 (JNK1) and JNK2 through Elk-1, controlling c-Jun expression and cell proliferation. Mol Cell Biol 27: 54-64, 2007.

79. Chow CW,Dong C,Flavell RA and Davis RJ: c-Jun NH(2)-terminal kinase inhibits targeting of the protein phosphatase calcineurin to NFATc1. Mol Cell Biol 20: 5227-5234, 2000.

80. McCall MN, Kent OA, Yu J, Fox-Talbot K, Zaiman AL and Halushka MK: MicroRNA profiling of diverse endothelial cell types. BMC Med Genomics 4: 78, 2011

(i) $($ This work is licensed under a Creative Commons Attribution-NonCommercial-NoDerivatives 4.0 International (CC BY-NC-ND 4.0) License. 